\title{
T Tauri stars: magnetic fields and planets
}

\author{
K.N. Grankin and the MaTYSSE collaboration
}

Crimean Astrophysical Observatory, Nauchny, Crimea, 298409

e-mail: konstantin.grankin@craocrimea.ru

Submitted on August 21, 2020

\begin{abstract}
In this short review we present the results of a study of the large-scale magnetic topologies of T Tauri stars (TTS). A small spectropolarimetric survey of 8 young stars was carried out within two international projects MaPP (Magnetic Protostars and Planets) and MaTYSSE (Magnetic Topologies of Young Stars and the Survival of massive close-in Exoplanets) between 2009 and 2016. For each of our targets we reconstructed the brightness map and the magnetic field topology using Zeeman-Doppler imaging (ZDI). This review contains a brief description of spectropolarimetric data, the ZDI method, one example of the reconstruction of brightness and magnetic maps, and the properties of magnetic fields of 8 TTS. Our results suggest that AA Tau and LkCa 15 interact with their disks in the propeller mode when their rotation is actively slowed by the star/disk magnetic coupling. We find that magnetic fields of some TTS are variable on a time scale of a few years and are thus intrinsically nonstationary. We report on the detection of a giant exoplanet around V830 Tau and TAP 26. These two new detections suggest that the type II disk migration is efficient at generating newborn hot Jupiters (hJs) around young TTS. The result of our survey is compared to the global picture of magnetic field properties of twenty TTS in the Hertzsprung-Russell diagram. The comparison shows that WTTS exhibit a wider range of field topologies as compared to CTTS, and that magnetic fields of all TTS (CTTS and WTTS as a whole) are mostly poloidal and axisymmetric when they are mostly convective and cooler than $4300 \mathrm{~K}$. This needs to be confirmed with a larger sample of stars.
\end{abstract}

Key words: young stars, magnetic fields, protoplanetary disks, exoplanets

\section{Introduction}

T Tauri stars (TTS) are young low-mass stars of late spectral types which are at the stage of gravitational contracting and have not reached the main-sequence (MS) yet. Some of these objects, "classical" TTS (CTTS), are surrounded by massive accretion gas and dust protoplanetary disks. Other TTS with weak emission lines (WTTS) do not show signatures for the presence of any disks at all or there are the so-called "transition" disks which have lost the dust component in inner regions due to the processes of accretion and formation of planetary systems.

The presence of magnetic fields on TTS to a great extent determines the character of the interaction between a star and its accretion disk, plays a crucial role in the evolution of the angular momentum of a contracting star, and can influence the formation and migration of young planets. Thus, studying properties of TTS magnetic fields is of primary importance for testing and developing stellar dynamo models and theories of the formation of solar-type stars and their planets. With the aim of studying the large-scale topology of TTS magnetic fields two international research programs MaPP (Magnetic Protostars and Planets) and MaTYSSE (Magnetic Topologies of Young Stars and the Survival of massive close-in Exoplan- ets) were organized. The first program was implemented in 2009-2012, the second one - in 2013-2016.

These two research programs have jointed efforts of scientists from France, Great Britain, Canada, USA, Taiwan, Brazil, Chile, Portugal, China, Italy, Switzerland, and astronomers from the Crimean Astrophysical Observatory. The most part of observations within the MaPP program were derived with the ESPaDOnS spectropolarimeter at the 3.6-m Canada-France-Hawaii Telescope (CFHT) located on the island of Hawaii. Within the second program MaTYSSE two more spectropolarimeters were additionally used: NARVAL at the 2-m Bernard Lyot Telescope (TBL) (Pic du Midi, France) and HARPS-Pol at the 3.6-m ESO telescope (La Silla, Chile). This review represents the most interesting results within the MaPP and MaTYSSE programs acquired with the direct participation of researchers from $\mathrm{CrAO}$.

\section{Spectropolarimetric data}

The large majority of spectral data on young stars were derived during one or two observational seasons. Each observing run had a duration of one to two weeks, and the spectral data covered from 5 to 10 stellar rotation cycles. Each spec- 
Table 1. Parameters of stars and spectral data

\begin{tabular}{llrrlccccccc}
\hline Star & type & $\begin{array}{c}T_{\text {eff }} \\
\mathrm{K}\end{array}$ & $\begin{array}{r}v \sin i \\
\mathrm{~km} \mathrm{~s}^{-1}\end{array}$ & $\begin{array}{l}P_{\text {rot }} \\
\text { days }\end{array}$ & $\begin{array}{c}\mathrm{M} \\
M_{\odot}\end{array}$ & $\begin{array}{c}\mathrm{t} \\
\text { million years }\end{array}$ & $\begin{array}{c}\text { epoch } \\
2000+\end{array}$ & $N_{S}$ & $N_{\text {obs }}$ & $S / N$ & $\overline{S / N}$ \\
\hline AA Tau & $\mathrm{c}$ & 4000 & 11.3 & 8.22 & 0.70 & 1.5 & $07-09$ & 2 & 24 & $100-200$ & 150 \\
V2129 Oph & $\mathrm{c}$ & 4500 & 14.5 & 6.53 & 1.35 & $2-3$ & 09 & 1 & 23 & $100-210$ & 180 \\
LkCa 4 & $\mathrm{w}$ & 4100 & 28.0 & 3.374 & 0.79 & 2 & 14 & 1 & 12 & $120-180$ & 160 \\
LkCa 15 & $\mathrm{c}$ & 4500 & 13.0 & 5.70 & 1.25 & 5 & 15 & 1 & 14 & $110-180$ & 160 \\
TAP 26 & $\mathrm{w}$ & 4620 & 68.2 & 0.7135 & 1.04 & 17 & $15-16$ & 2 & 29 & $100-150$ & 140 \\
V819 Tau & $\mathrm{w}$ & 4250 & 9.5 & 5.5311 & 1.00 & 3.2 & $14-15$ & 1 & 30 & $140-220$ & 190 \\
V830 Tau & $\mathrm{w}$ & 4250 & 30.5 & 2.7410 & 1.00 & 2.2 & $14-16$ & 2 & 56 & $80-170$ & 150 \\
V410 Tau & $\mathrm{w}$ & 4500 & 73.2 & 1.87197 & 1.42 & 0.8 & $08-16$ & 4 & 129 & $82-238$ & 140 \\
\hline
\end{tabular}

trum generally covered the whole optical range (from 370 to $1000 \mathrm{~nm}$ ) with a resolution of 65000 (i.e., $4.6 \mathrm{~km} \mathrm{~s}^{-1}$ ) and a spectral sampling of $2.6 \mathrm{~km} \mathrm{~s}^{-1}$. Both the nonpolarized (the $I$ Stokes parameter) and polarized spectra (the $V$ Stokes parameter) were derived for each object. All the polarized spectra are composed of four separate sub-exposures acquired at different positions of the polarimeter, which allows one to take into account the instrumental polarization effects of the first order.

All the input spectra were processed with the data reduction package Libre Esprit available at the CFHT and TBL telescopes. This package automatically performs an optimal extraction of nonpolarized and polarized spectra. Owing to the fact that the spectrograph slit has an inclination with respect to the CCD lines, the spectral orders that correspond to different CCD lines have different samples of pixels. The Libre Esprit package uses this possibility for optimal extraction of spectra with denser sampling than the initial CCD sampling. As a result, a spectral resolution of about $1.8 \mathrm{~km}$ $\mathrm{s}^{-1}$ is achieved. All the spectra are automatically corrected for spectral shifts resulting from instrumental effects (for instance, due to mechanical bendings, fluctuations of temperature and pressure). For this aim, the telluric lines are used as a standard. Though these procedures of the Libre Esprit package are not ideal, they ensure an accuracy in determining radial velocity $(\mathrm{RV})$ of better than $30 \mathrm{~m} \mathrm{~s}^{-1}$.

The peak signal-to-noise ratios reached in the derived spectra are in the range from 70 to 90 for the NARVAL data and from 100 to 220 for theESPaDOnS data. The basic information on stars and derived spectral data is summarized in Table 1, where we note the name of a star, its type ("c" if CTTS, or " $\mathrm{w}$ " if WTTS), effective temperature, $v \sin i$ value, rotation period, mass, age, epoch of spectral observations, the number of observational seasons $\left(N_{S}\right)$, the number of spectra, the range of values of the signal-to-noise ratio $(S / N)$, and the average value of the signal-to-noise ratio $(\overline{S / N})$.

\section{Doppler-Zeeman imaging}

To analyze spectral data, the Least-Squares Deconvolution technique (LSD) was used. This technique of mutual correlation makes it possible to calculate the mean profile of the pseudoline from several thousand spectral lines which behave similarly in the magnetic field. The main advantage of the LSD technique is a substantial increase of the resultant $\mathrm{S} / \mathrm{N}$ when constructing both $V$ and $I$ pseudoline profiles. The list of lines used for calculating the mean LSD local profile was formed on the basis of the atmosphere model under the conditions of local thermodynamic equilibrium ATLAS9 for a certain spectral type and value of $\log g$, which correspond to a certain studied star. Only moderate or strong atomic spectral lines forming in the stellar photosphere were included in this list. As a rule, when calculating the mean LSD local profile, there were used from seven to nine thousand spectral lines, whereas about $40 \%$ of them are the Fe I lines. As a result, the $\mathrm{S} / \mathrm{N}$ for the mean LSD I profile reached the values from 950 to 3000 for a velocity bin of $1.8 \mathrm{~km} \mathrm{~s}^{-1}$. The $\mathrm{S} / \mathrm{N}$ for the LSD $V$ profile, where prevails the noise of photon statistics, varied in the range from 1800 to 7000 depending on object's brightness and weather conditions.

In order to reconstruct brightness distribution over the stellar surface and reconstruct the magnetic field topology, we have used the tomographic method of Doppler-Zeeman imaging (ZDI). This method allows us to transform the simultaneous time series of one-dimensional LSD $I$ and $V$ profiles into two-dimensional images: the surface brightness map (brightness map) and the magnetic field map (magnetic map). From the mathematical point of view, ZDI follows the principles of reconstructing images by the maximum entropy method and iteratively searches for an image with the least information content, which corresponds to the certain given level $\chi^{2}$. The surface magnetic field is modeled as a combination of the poloidal and toroidal fields represented as the weighted sums of spherical harmonics (with orders of $l=1$ to $l=15$ ) which are projected onto the spherical coordinate space (Donati et al., 2010).

In the modified version of the ZDI method the reconstructed brightness map allows one to reveal not only dark spots but bright areas. This is achieved due to the fact that the reconstructed surface brightness (normalized with respect to the photosphere brightness) can change within the range both lower and higher than unity but is not restricted by the interval $[0,1]$, as it was earlier. It is worth noting that the detected bright areas are attributed to the photosphere region that is brighter than the quiet photosphere rather than to the bright region at the chromospheric level, as in the case of the Sun (Donati et al., 2014).

To model local Stokes $I$ and $V$ LSD profiles, we used the Unno-Rachkovsky equations for transferring polarized radiation in the Milne-Eddington atmosphere model taking into account local brightness and magnetic field. Then these local profiles were integrated over the apparent stellar hemisphere in order to acquire synthetic mean photospheric LSD 
profiles for their further comparison with our spectral observations. Such a calculation method ensures, in particular, a reliable description of how line profiles are distorted in the presence of magnetic fields. The basic parameters of the local LSD profile used in the ZDI method are as follows: the central wavelength of a pseudoline is $670 \mathrm{~nm}$, the Doppler line width $-1.8 \mathrm{~km} \mathrm{~s}^{-1}$, the equivalent width $-3.9 \mathrm{~km} \mathrm{~s}^{-1}$, and the Lande factor -1.2 .

Calculating a value of the latitudinal shift that affects brightness maps during the time, the ZDI method allows one to estimate differential rotation at the photospheric level. An account of differential rotation contributes to purer filtration of input RV curves and makes it possible to perform a search for the signal from a planet in residual RV curves. To acquire absolute veiling estimates in the case of analyzing spectra of CTTS, a standard star was used with analogous temperature and luminosity class (as a rule, WTTS) observed with the same spectropolarimeter. A spectrum of the standard star was artificially broadened up to the same value of $v \sin i$, veiled, and cross-correlated with each spectrum of the studied CTTS for achieving the best fit. This procedure made it possible to simultaneously take into account the veiling and determine the radial velocity (Alencar et al., 2018).

\section{Reconstruction of brightness and magnetic maps}

As an example, in Fig. 1 we represent a set of Stokes $I$ and $V$ LSD profiles obtained for $\mathrm{LkCa} 4$ during January 2014 along with a model fitting of these data. The resultant fitting corresponds to the minimal value of $\chi^{2}=1$. At the beginning of this fitting procedure the value of $\chi^{2}$ was more than 50 , which corresponded to the zero magnetic field and the brightness map without large temperature inhomogeneities. In Fig. 1 one can see a high quality of both the set of spectral data itself and the resultant fitting. Taking into consideration this fact, the high value of $v \sin i=28.0 \pm 0.5 \mathrm{~km} \mathrm{~s}^{-1}$, and a rather dense phase coverage, one can confidently claims that any significant random artifacts or shifts should not be expected.

Figure 2 shows the resultant maps reconstructed from the set of observed $I$ and $V$ profiles using the ZDI method. The basic property of the brightness map (top left panel in Fig. 2 ) is a dark spot of complex shape near the polar region. This spot proved to be nonaxisymmetric, with several appendages toward lower latitudes, as evidenced by a significant variation in nonpolarized LSD profiles (left panel in Fig. 1). The second most conspicuous feature of the brightness map is the presence of a bright area located at middle latitudes near the phase 0.42 . The brightness of this area is by $50 \%$ higher than the mean brightness of the surrounding quiet photosphere. The dark spot and the bright region cover in total $25 \%$ of the whole stellar surface. It should be noted that the photometric brightness variations predicted by the reconstructed brightness map are in very good agreement with photometric observations derived at $\mathrm{CrAO}$ throughout four months. This testifies to the fact that the lifetime of brightness elements on the surface of $\mathrm{LkCa} 4$ exceeds a few months, which is fully consistent with previous conclusions from the longterm photometric monitoring showing that the light curves
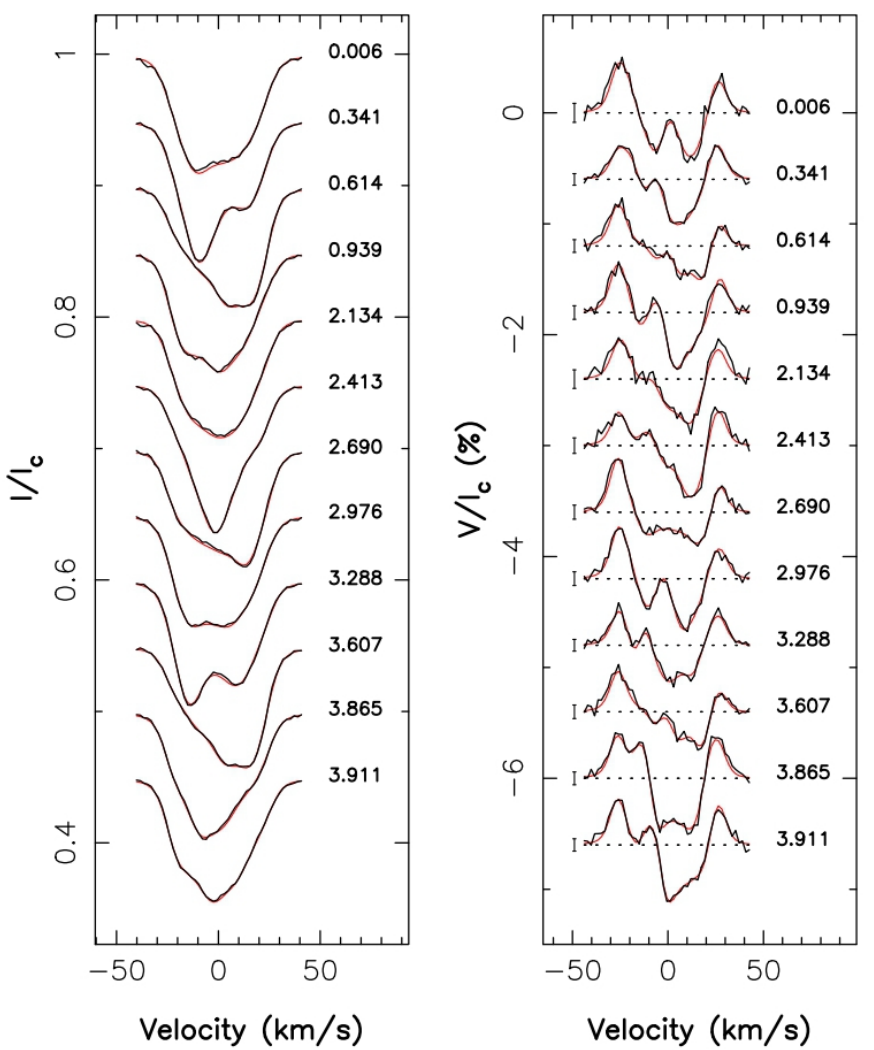

Fig. 1. The set of LSD profiles for LkCa 4 acquired in January 2014. Nonpolarized (I Stokes parameter) and polarized ( $V$ Stokes parameter) LSD profiles are shown in the left and right panels, respectively. Observed profiles are marked by the black line, the results of fitting by the maximum entropy method - by the thin red line. Rotational cycles and error bars (for the $V$ Stokes profile) are shown near each profile (Fig. 3 from the paper of Donati et al., 2014)

of this star are remarkably stable over time in both shape and phase (see Fig. 5 in the paper of Grankin et al. (2008)).

The magnetic map demonstrates a quite simple largescale magnetic structure composed of two basic components. The first component is an axisymmetric poloidal field comprising up to $70 \%$ of magnetic energy. Whereas $94 \%$ of the poloidal field energy is concentrated in the modes of spherical harmonics (SH) with $m<l / 2$ ( $l$ and $m$ denote degrees and mode orders, respectively), and $86 \%$ of this energy is contained in the dipole component $(l=1$ and $m=0)$. The poloidal component can be approximated by the dominating dipole $(1.6 \mathrm{kG})$ inclined at an angle of $\simeq 10^{\circ}$ to the rotation axis (toward the phase 0.75 ) and by the 4 times weaker $(\simeq 0.4 \mathrm{kG})$ axisymmetric octupole component. This results in the generation of the intensive radial field, which exceeds 2 $\mathrm{kG}$ in the visible stellar pole of the star (see the top right panel in Fig. 2) and coincides with the dark spot reconstructed in the brightness map. Note also that the elongated shape of this magnetic pole is similar to the shape of the dark polar spot.

The second basic component of the large-scale field of this star is a very significant, almost axisymmetric toroidal component manifesting as a ring of the azimuthal field $(\simeq 1$ 

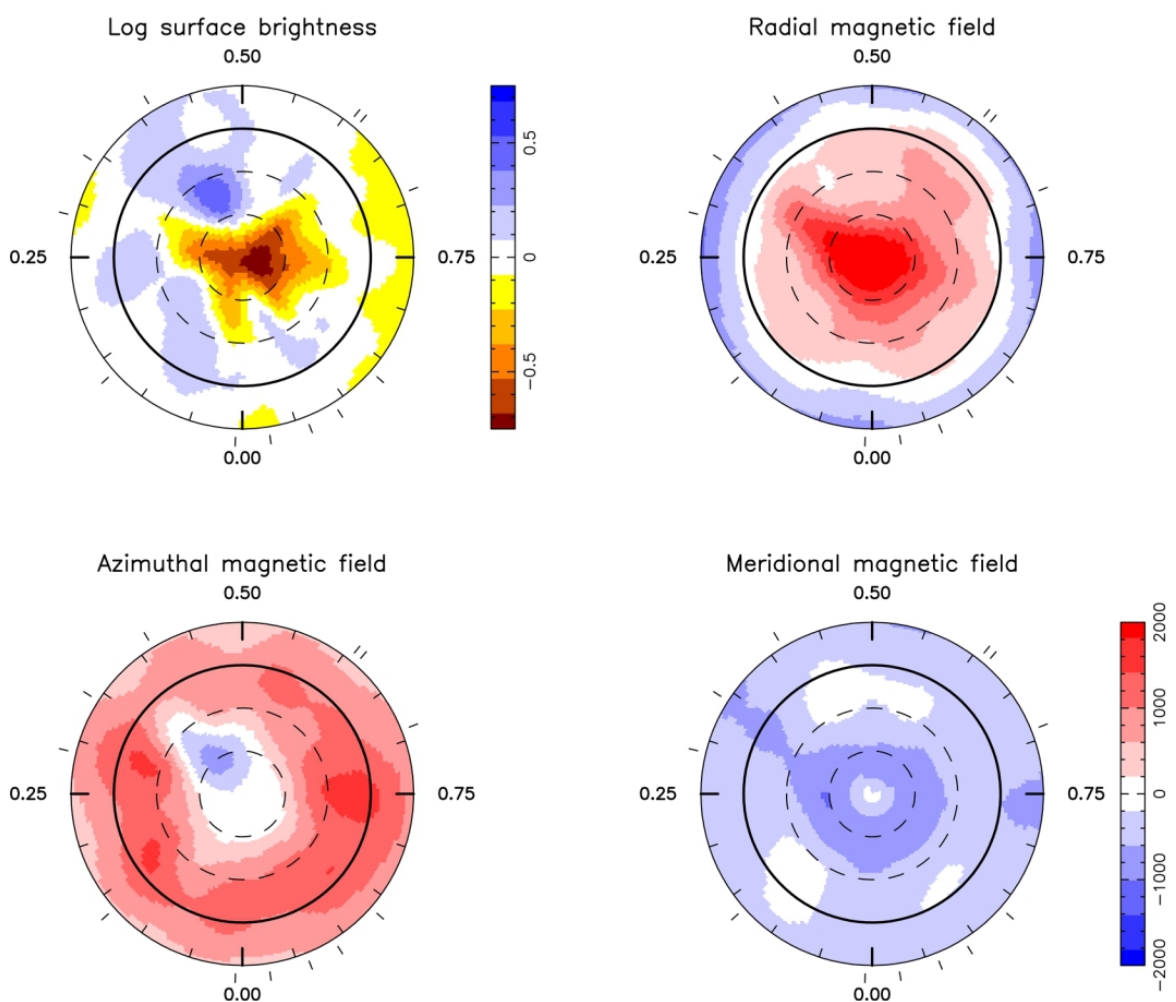

Fig. 2. Maps of logarithmic brightness (the relatively quiet photosphere, top left panel), radial (top right panel), azimuthal (bottom left panel), and meridional (bottom right panel) magnetic field components on the surface of LkCa 4 in January 2014. Magnetic fluxes are marked with color from -2000 to $2000 \mathrm{G}$. In all the panels the stellar surface is represented in the flat polar projection up to the latitude $-30^{\circ}$. The equator is shown as a thick circle, while parallels are marked with dashed circles. The radial dashes around each image indicate phases of spectral observations (Fig. 4 from the paper of Donati et al., 2014)

$\mathrm{kG}$ ) which surrounds the star at equatorial latitudes (see the bottom left panel in Fig. 2). This feature directly reflects an almost symmetric shape of $V$ LSD profiles profiles (near the center of the line profile) at most rotation phases (see the right panel in Fig. 1), which, as known, is a distinct signature of the visible ring of the azimuthal field on the stellar surface (Donati et al., 2014). We also note the detection of a local feature of the azimuthal field of opposite polarity (clockwise) in close proximity to the bright area detected in the brightness map. Spherical harmonics that describe the reconstructed field are restricted by terms with $l \leq 10$. Only insufficient variations in the solution are observed at increasing maximum value $l$ from 10 to 15 , which evidences that the most part of the signal detected in $V$ LSD profiles of $\mathrm{LkCa} 4$ is concentrated on larger spatial scales.

\section{Results of Doppler-Zeeman imaging}

Figure 3 and Fig. 4 represent brightness and magnetic maps for 3 CTTS and 3 WTTS reproduced from the spectropolarimetric data derived within two international projects MaPP and MaTYSSE with the direct participation of researchers from CrAO.

AA Tau. In the brightness map there is a reconstruction of the elongated spotted region of complex configuration which extends to almost $180^{\circ}$ in the latitudinal direction (Fig. 3, top left panel). The darkest zone of this region is at a latitude of about $50^{\circ}$ and almost coincides with the most intensive region of the radial magnetic field component. The magnetic field contains a significant axisymmetric poloidal component which concentrates $80-85 \%$ of magnetic energy on the stellar surface. In its turn, about $90-95 \%$ of the poloidal component's energy is concentrated in the dipole component ( $l=1$ and $m=0)$. In fact, the poloidal component can be almost fully approximated by the dominating dipole $(\simeq 2 \mathrm{kG})$ inclined at about $20^{\circ}$ to the rotation axis (toward the phase 0.20) (Fig. 3, middle top panel). It should be noted that the octupole component of the poloidal field is at least 5 times less than the dipole component. This circumstance makes AA Tau very similar to $M$ dwarfs, whose large-scale fields are also strong, generally poloidal and axisymmetric (Morin et al., 2008). We also find out that the field has a pronounced toroidal component which contains about 15-20\% of the whole magnetic energy. This component manifests itself in the form of fragments of the equatorial ring of the negative (in a clockwise direction) azimuthal field with a strength of 0.5-1 kG (Fig. 3, top right panel).

The matter accretion rate of the protoplanetary disk onto the surface of AA Tau estimated from the emission in the He I $D_{3}$ line is variable in the range from $10^{-9.6}$ to $10^{-8.5} M_{\odot} \mathrm{yr}^{-1}$ and on average is equal to $10^{-9.2} M_{\odot} \mathrm{yr}^{-1}$. This is by an order of magnitude lower than the accretion rate at which the Alfven radius (lower than which the disk is destroyed by the stellar 

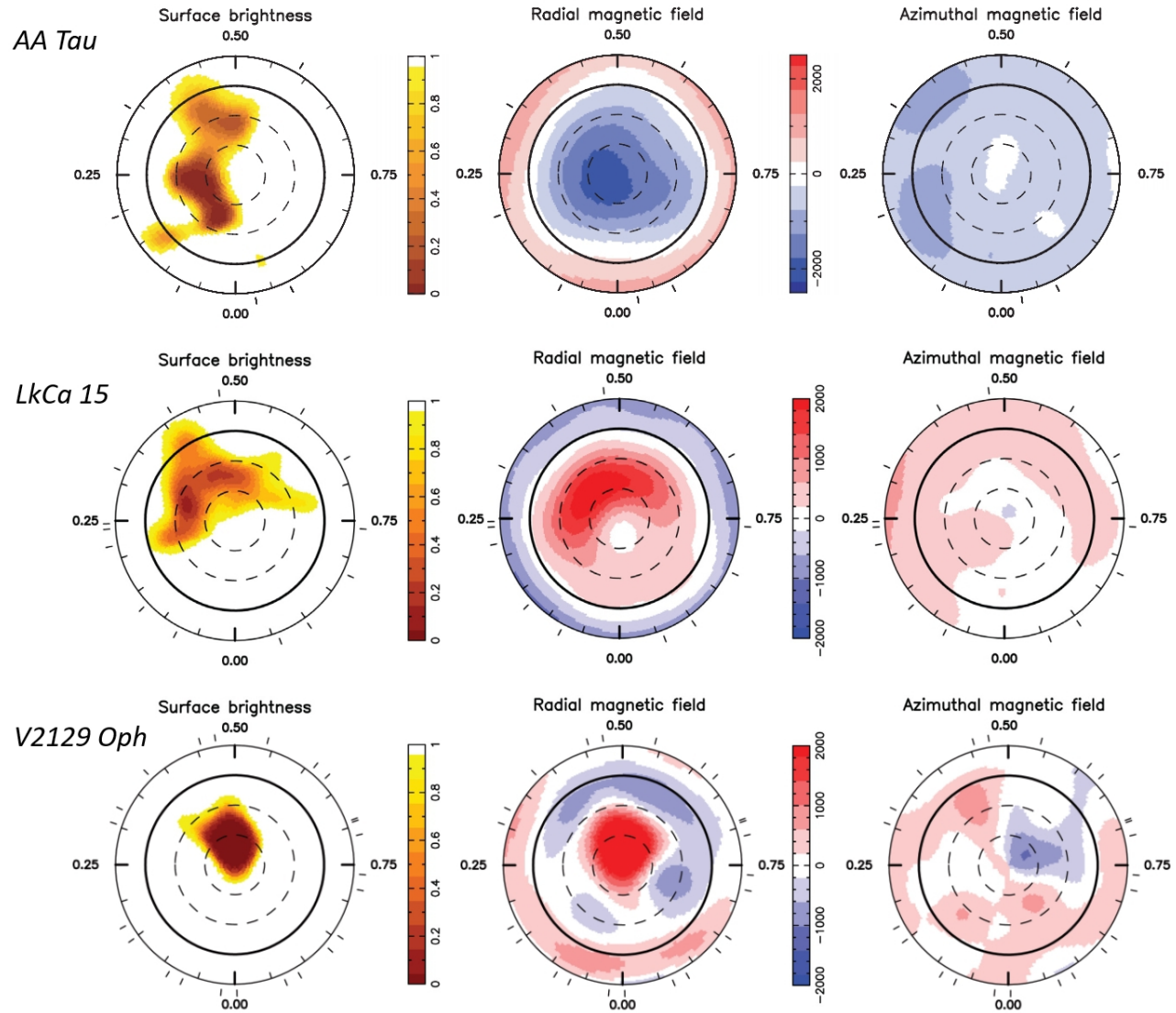

Fig. 3. Maps of brightness (left panel), radial (middle panel), and azimuthal (right panel) magnetic field components on the surface of three CTTS: AA Tau (at the end of 2007), LkCa 15 (in November 2015), and V2129 Oph (in July 2009). Notation is similar to that in Fig. 2. This figure is constructed on the basis of Fig. 5 from Donati et al. (2010), Fig. 3 from Donati et al. (2019), and Fig. 6 from Donati et al. (2011)

magnetic field) is equal to the corotation radius (at which the Kepler orbital period is equal to the stellar rotation period) and at which accretion is possible. This means that AA Tau is at the propeller mode when the most part of accreting matter is ejected outward and only a small part falls on the stellar surface. Thus, the rotation of AA Tau effectively slows down because of a magnetic relation between the star and the protoplanetary disk (Donati et al., 2010).

LkCa 15. The magnetic map of this star shows a region of the strong radial field at intermediate latitudes $\left(30-60^{\circ}\right)$ that is significantly elongated in the longitudinal direction and centered on the phase 0.4 where the field strength reaches $2.2 \mathrm{kG}$. This region of the radial field is overlapped with the extended spotted region which extends to almost $180^{\circ}$ in the longitudinal direction and goes to the equator (and even lower) at the phase 0.4 (Fig. 3, left middle panel). We find that the magnetic field has a strong axisymmetric poloidal component containing up to $85 \%$ of magnetic energy, as in the case of AA Tau. This poloidal component is generally composed of a dipole $(1.35 \mathrm{kG})$ inclined at an angle of $\simeq 20^{\circ}$ to the rotation axis (toward the phase 0.40 ), which contains $\simeq 75 \%$ of the poloidal field energy. The poloidal field also includes the octupole component of opposite sign $(-0.9 \mathrm{kG})$, which accumulates $\simeq 20 \%$ of the poloidal field energy (Donati et al., 2019).

The average rate of the disk matter accretion onto the surface of LkCa 15 accounts for $10^{-9.2} M_{\odot} \mathrm{yr}^{-1}$. This means that the strong magnetic field of $\mathrm{LkCa} 15$ is able to purify the inner regions of the protoplanetary disk up to the distance 0.07 a.u., at which the Kepler orbital period is equal to the stellar rotation period. Our results show that $\mathrm{LkCa} 15$, as well as AA Tau, is in the propeller mode in the course of which there occurs an effective rotation slow-down of the young star by a strong dipole component of the magnetic field.

V2129 Oph. The reconstructed large-scale topology of the magnetic field of V2129 Oph in July 2009 is almost fully poloidal: only $5 \%$ of magnetic energy is contained in the toroidal component. Contrary to AA Tau and $\mathrm{LkCa}$ 15 , the poloidal field of V2129 Oph is not axisymmetric, whereas the octupole component is 2-3 times stronger than the dipole. The maximum strengths of the oclupole and dipole components account for about 2.1 and $0.9 \mathrm{kG}$, respectively. The general structure that is reconstructed is the intensive region of the radial field at high latitudes where the radial filed strength reaches $4 \mathrm{kG}$ (Fig. 3, bottom middle panel). This region coincides with the dark spot which is reconstructed in 

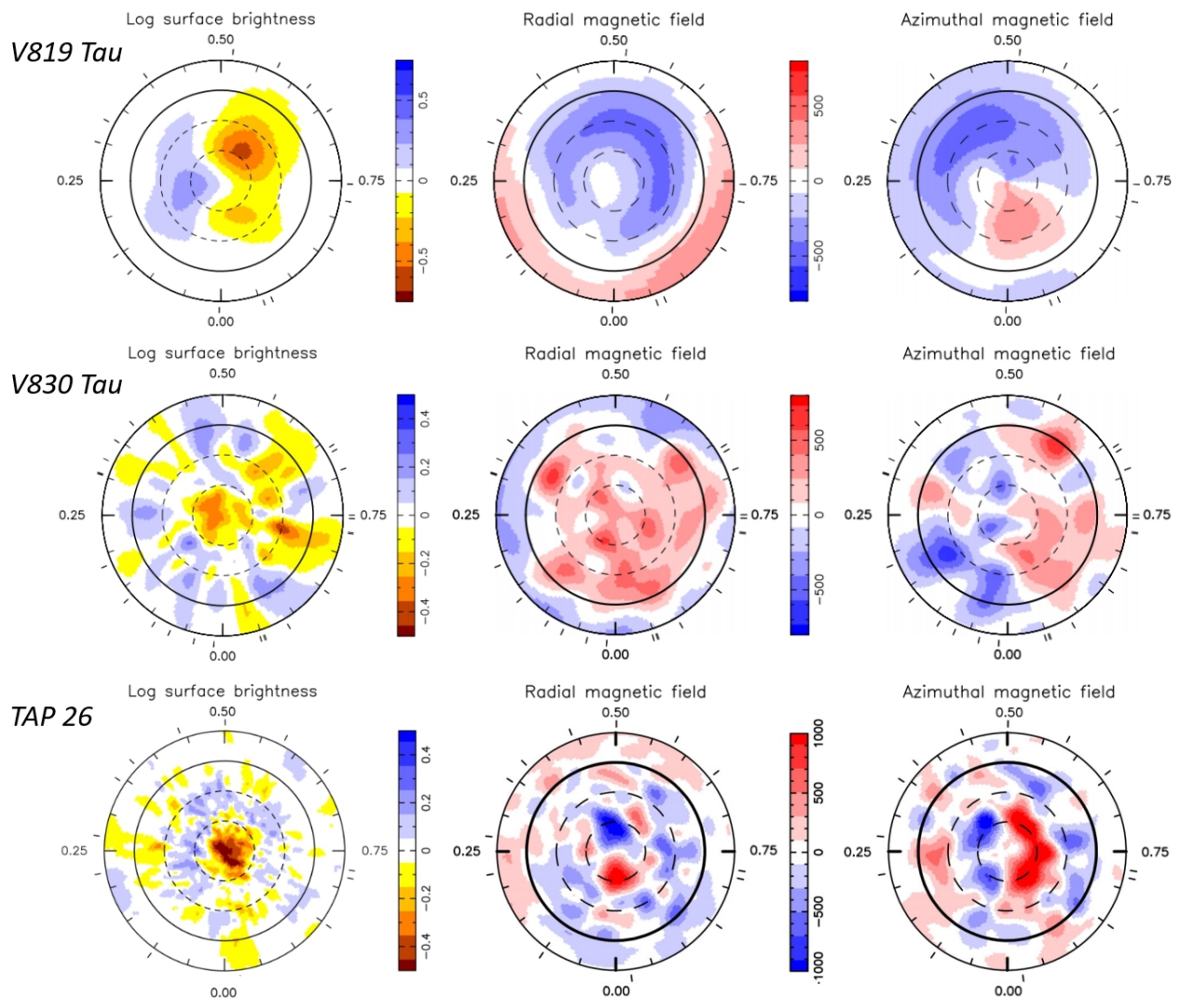

Fig. 4. Maps of logarithmic brightness (left panel), radial (middle panel), and azimuthal (right panel) magnetic field components on the surface of three WTTS: V819 Tau (in December 2014 - January 2015), V830 Tau (in January - February 2016), and TAP 26 (in January 2016). Notation is similar to that in Fig. 2. This figure is constructed based on Fig. 4 and Fig. 6 from Donati et al. (2015), Fig. 3 and Fig. 5 from Donati et al. (2017), and Fig. 3 and Fig. 5 from Yu et al. (2017)

the brightness map (Fig. 3, bottom left panel). The octupole component of the poloidal field is directly followed as a fragmented equatorial ring of the negative radial field which surrounds the general high-latitude region of the radial field (Donati et al., 2011).

A comparison of this field configuration reproduced from our observations in 2009 with configuration of the field in 2005 reconstructed in the earlier paper of Donati et al. (2007) showed that it can undergo significant changes. We detected that the dipole component increased by almost a factor of 3.5 (from 280 to $970 \mathrm{G}$ ) as compared to 2005 , whereas the octupole component increased by a factor of 1.3 (from 1620 to $2160 \mathrm{G}$ ). Meanwhile, the ratio of intensity of the octupole component to that of the dipole one decreased by a factor of 2.6, approximately from 5.8 (in 2005) to 2.2 (in 2009). This result implies that the magnetic fields of young stars can be variable on a time scale of several years and thus are nonstationary. Such an assumption was brilliantly confirmed from the analysis of our many-year spectral observations of V410 Tau which will be discussed below.

V819 Tau. The brightness map of this star (see Fig. 4, top left panel) resembles the map of $\mathrm{LkCa} 4$ (see Fig. 2, top left panel) with the dark spot near the pole. A light area of less contrast is next to it. A lower spatial resolution on the surface of V819 Tau as compared to $\mathrm{LkCa} 4$ is associated with a slower rotation of this $\operatorname{star}\left(v \sin i=9.5 \pm 0.5 \mathrm{~km} \mathrm{~s}^{-1}\right)$, in the case of LkCa 4 we have $v \sin i=28.0 \pm 0.5 \mathrm{~km} \mathrm{~s}^{-1}$. A cool spot covers $9 \%$ of the whole surface, while a warm one $-6 \%$. It is worth noting that these estimates of spot areas should be considered only as lower limits. The point is that the ZDI method is effective for the reconstruction of only large-scale formations which cause the detected distortions of the line profile that is insensitive to small structures more or less uniformly distributed over the stellar surface (Donati et al., 2015).

The magnetic field is much weaker than that on $\mathrm{LkCa}$ 4 and is generally poloidal ( $80 \%$ of magnetic energy) with a mean value of $370 \mathrm{G}$. The most part of the poloidal field is concentrated in the dipole component $(75 \%)$. In the first order and at some distance from the star the poloidal field of V819 Tau can be approximated by the dipole (400 G) inclined at an angle of $30^{\circ}$ to the rotation axis toward the phase 0.65 (see Fig. 4, top middle panel). A weaker octupole component of the poloidal field $(100-150 \mathrm{G})$ is more or less aligned on the rotation axis and opposite in sign to the dipole component. The large-scale field of V819 Tau also includes a weak toroidal component $(170 \mathrm{G})$ whose topology is more complicated and less axisymmetric than that for $\mathrm{LkCa}$ 4 (Fig. 4, top right panel). 
Table 2. Parameters of ZDI models for V410 Tau

\begin{tabular}{|c|r|c|c|c|c|c|c|c|c|c|c|c|c|c|}
\hline Year & $N_{\text {obs }}$ & $\begin{array}{c}\text { spots } \\
\text { dark }\end{array}$ & $\begin{array}{c}\text { spots } \\
\text { bright }\end{array}$ & $\begin{array}{c}B \\
(\mathrm{G})\end{array}$ & $r_{\text {pol }}$ & $r_{\text {tor }}$ & $r_{\text {d/pol }}$ & $r_{\text {s/pol }}$ & $r_{\text {d/tor }}$ & $r_{\text {s/tor }}$ & $\begin{array}{c}B_{\text {dip }} \\
(\mathrm{G})\end{array}$ & $\begin{array}{c}\text { inclination } \\
\text { dipole }\end{array}$ & phase & $\begin{array}{c}R V \\
\left(\mathrm{~km} s^{-1}\right)\end{array}$ \\
\hline$(1)$ & $(2)$ & $(3)$ & $(4)$ & $(5)$ & $(6)$ & $(7)$ & $(8)$ & $(9)$ & $(10)$ & $(11)$ & $(12)$ & $(13)$ & $(14)$ & $(15)$ \\
\hline 2008 & 6 & 5.8 & 4.4 & 486 & 0.32 & 0.68 & 0.13 & 0.37 & 0.89 & 0.96 & 129 & 23 & 0.71 & 16.30 \\
2009 & 48 & 9.6 & 7.1 & 556 & 0.55 & 0.45 & 0.26 & 0.09 & 0.54 & 0.79 & 165 & 54 & 0.54 & 16.30 \\
2011 & 20 & 8.1 & 6.6 & 560 & 0.40 & 0.60 & 0.24 & 0.23 & 0.72 & 0.85 & 239 & 44 & 0.62 & 16.40 \\
2013 & 25 & 11.0 & 7.5 & 568 & 0.49 & 0.51 & 0.23 & 0.34 & 0.66 & 0.81 & 254 & 18 & 0.56 & 16.50 \\
2015 & 21 & 8.9 & 6.7 & 600 & 0.68 & 0.32 & 0.37 & 0.45 & 0.62 & 0.78 & 458 & 30 & 0.54 & 16.65 \\
2016 & 9 & 7.9 & 6.5 & 480 & 0.77 & 0.23 & 0.38 & 0.30 & 0.68 & 0.87 & 400 & 44 & 0.51 & 16.65 \\
\hline
\end{tabular}

V830 Tau. The brightness map of V830 Tau has a more complex structure as compared to the analogous map of V819 Tau (Fig. 4, middle left panel). The lower limits for the area of cold spots and warm areas account for $7 \%$ and $6 \%$, respectively. It should be noted that apart from the large cool polar spot there are several extended regions that go down to the equator and lower (Donati et al., 2017).

The magnetic field of V830 Tau is generally poloidal (90\% of magnetic energy) with a mean value of $350 \mathrm{G}$. The most part of the poloidal field is concentrated in the dipole component $(60 \%)$. In the first order and at some distance from the star the poloidal field of V830 Tau can be approximated by the dipole $(340 \mathrm{G})$ inclined at an angle of $22^{\circ}$ to the rotation axis toward the phase 0.88 . The octupole component of the poloidal field is weak $(150 \mathrm{G})$ and nonaxisymmetric. The large-scale field of V830 Tau also includes a weak toroidal component whose topology is more complicated than that for LkCa 4 and V819 Tau (Fig. 4, middle right panel).

TAP 26. The brightness map of TAP 26 reconstructed from our spectral observations in January 2016 is given in Fig. 4 in the bottom left panel. Apart from the coolest spot located in the polar region, there are a large number of small dark spots on the stellar surface which alternate with similar warm areas. Among dark low-latitude formations one can distinguish two low-latitude spotted regions located at phases 0.27 and 0.97 . Cool spots cover not less than $7 \%$ of the whole surface, while warm ones - not less than $5 \%$. The average strength of the large-scale magnetic field is about $430 \mathrm{G}$. The field is generally poloidal (up to $70 \%$ of magnetic energy), though it has a significant toroidal component (up to $30 \%$ ). The field is to a great extent axisymmetric, particularly in the case of the toroidal component (of $80 \%$ ). The poloidal component is axisymmetric only for $50 \%$ (Yu et al., 2017). The dipole component $(120 \mathrm{G})$ concentrates only $10 \%$ of the poloidal field energy, whereas the octupole and quadrupole modes accumulate up to $90 \%$ of the poloidal field energy.

\section{Nonstationary magnetic fields}

Among the stars of our sample V410 Tau is of particular interest due to peculiarities of its photometric behavior. Almost two decades of our photometric observations this star demonstrated a very stable phase light curve. During 1986-2004, variations in the brightness minimum phase did not exceed $0.16 P_{\text {rot }}$, where $P_{\text {rot }}$ is the rotation period (see Fig. 3 in the paper of Grankin et al., 2008). Meanwhile, the amplitude of the light curve varied within $0 .^{m} 25$ and $0 .^{m} 63$ in the $V$ band. Similar photometric behavior is characteristic of several most active and young WTTS, as well as of LkCa 4 (Grankin, 2013). However, in 2004-2006, the phase light curve started to evolve and about the phase 0.9 there was the second small maximum. In 2007, the variability amplitude achieved its record minimum $\left(0 .^{m} 05\right)$ at the almost constant mean brightness level (see Fig. 2 in the paper of Grankin, Artemenko, 2009). According to our photometric model, an almost complete disappearance of the periodic process in 2007 was associated with the fact that dark spots ceased to be concentrated at a certain longitude and were almost uniformly distributed over the stellar surface. Since a variation in the distribution of spots over the stellar surface reflects variations in the magnetic field structure, there are good reasons to say that the large-scale magnetic field topology of V410 Tau underwent important changes in 2006-2008. The first spectropolarimetric observations of this star undertaken by us in 2008 showed that on the stellar surface there were both a polar spot and a few low-latitude spots distributed quite uniformly in the longitudinal direction (Skelly et al., 2010), which was predicted in our earlier papers.

To investigate the magnetic field of V410 Tau and follow a possible evolution of its basic parameters over time, we undertook long-term spectropolarimetric observations of this star. Our set of spectropolarimetric data covers the time period between October 2008 and January 2016. (see the last line in Table 1). The full journal of observations is represented in the paper of Yu et al. (2019). The results of reconstructing brightness and magnetic maps acquired throughout six seasons are shown in Fig. 5. Brightness maps demonstrate a complex structure with a large number of relatively small and contrast details. A high concentration of dark spots near the pole is characteristic of all the maps. In January 2009, December 2013, and December 2015, the maps show a large contrast dark spot in the region of the equator located at phases $0.27,0.48$, and 0.48 , respectively. Remnants of this spot (spotted region) are observed in the map, which corresponds to January 2016, where its intensity became lower. A less contrast but more extended and complex in shape spotted equatorial region can be traced in the range of phases $0.6-0.7$ in January 2009. In January 2011, it splits into the regions with average phases 0.6 and 0.75 , whereas in 2013-2016 it is in the range of phases $0.75-0.90$. The main photometric brightness minimum falls on these phases in 2013-2016 (see Fig. 6). The phase light curves confirm the conclusions which were drawn as a result of the analysis of spectropolarimetric data using ZDI.

Parameters of ZDI models for each observational season are given in Table 2. The content of columns is as follows: (1) 


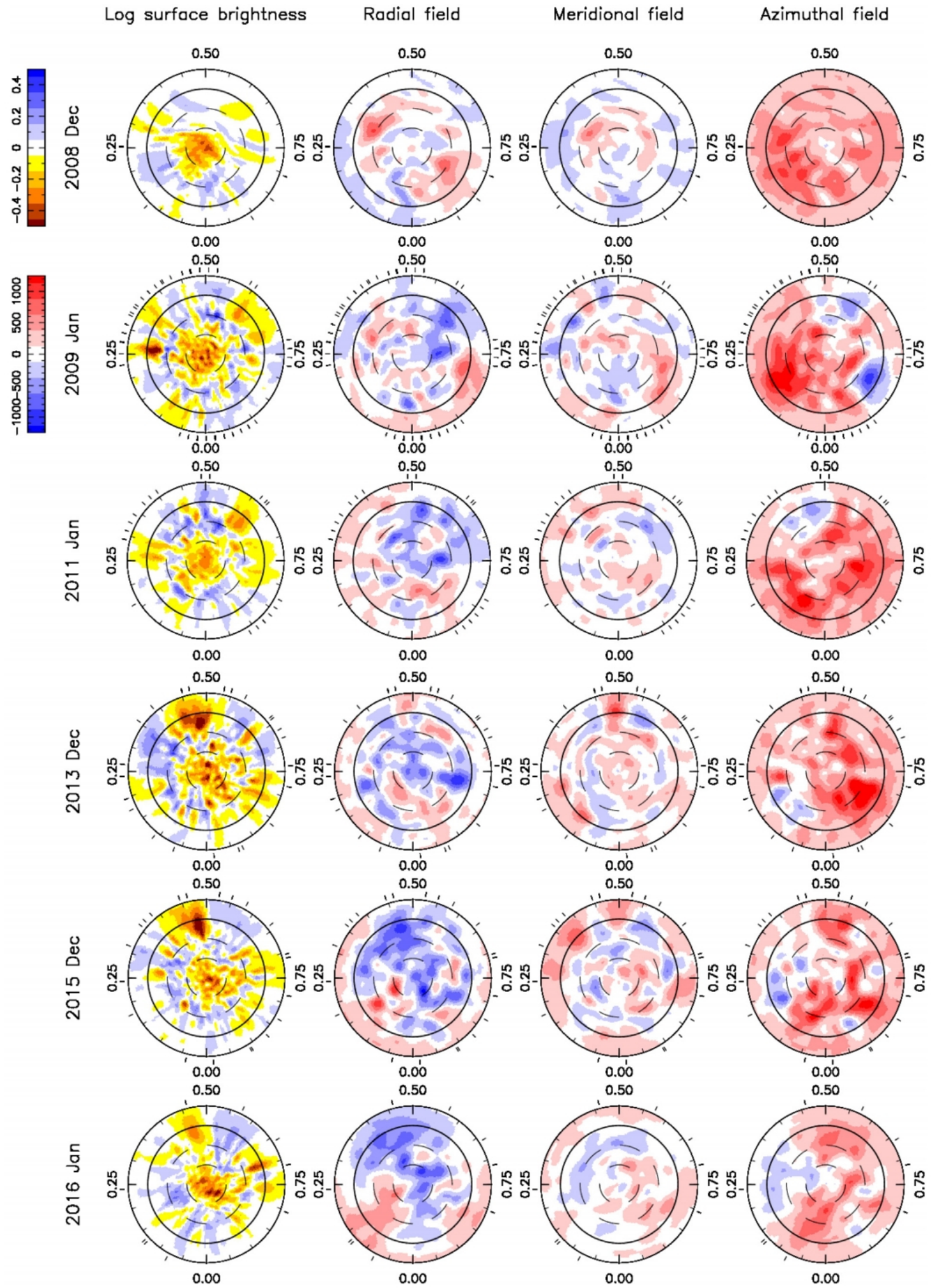

Fig. 5. Logarithmic maps of relative brightness (left column) and radial, meridional, and azimuthal magnetic field components (the second - fourth columns) on the surface of V410 Tau reconstructed from data derived in 2008-2016 (lines downward). Notation is similar to that in Fig. 2. (Fig. 3 from the paper of Yu et al., 2019) 

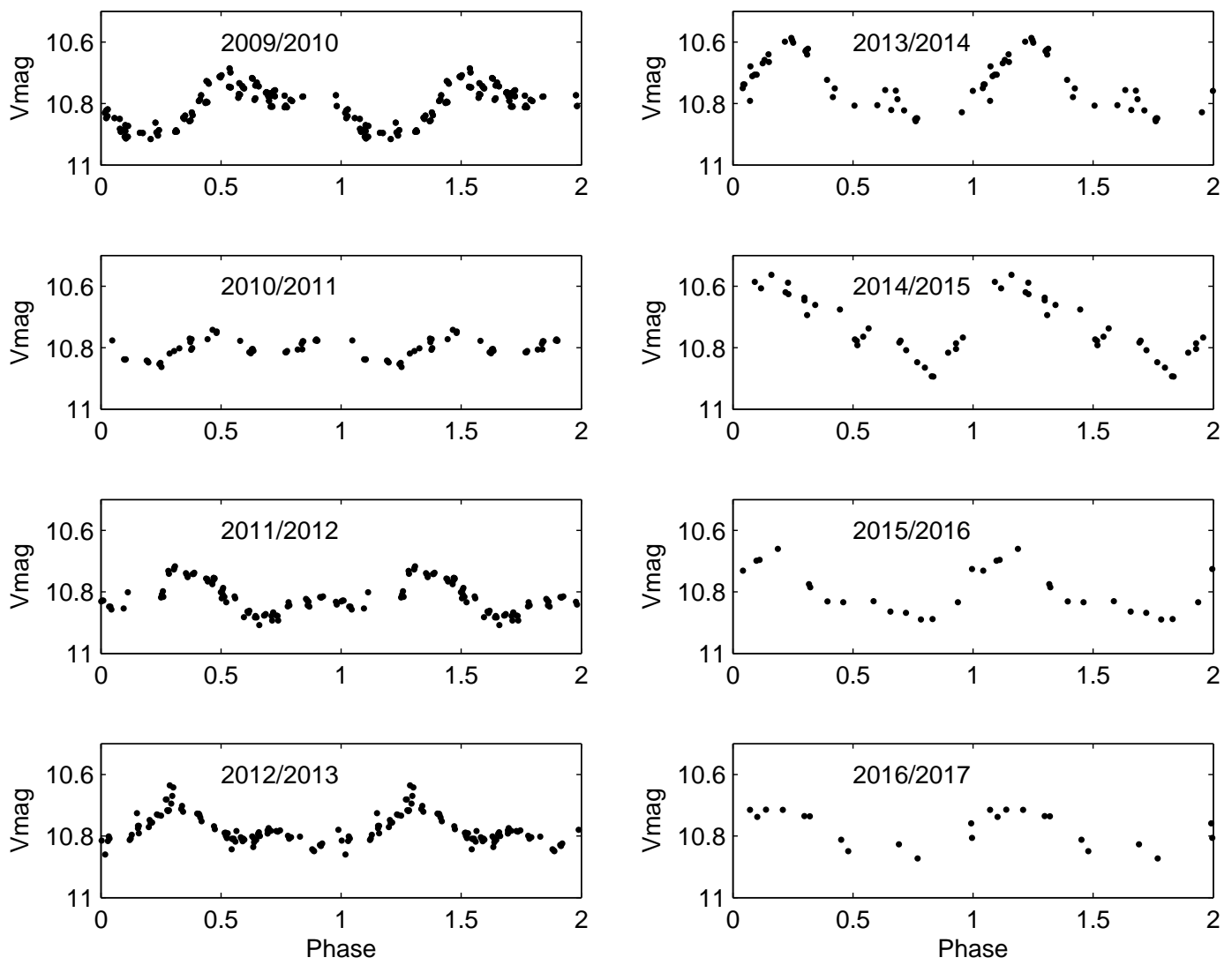

Fig. 6. Seasonal phase light curves of V410 Tau in the $V$ band derived in the course of spectral monitoring in 2009-2016. Variations in shape and amplitude of the light curve are caused by variations in both spot areas and distribution of spots over the surface

observational season; (2) number of spectral data; (3) relative areas of dark and (4) bright spots as a percentage of the visible stellar surface; (5) average strength of the magnetic field; (6) normalized contribution of the poloidal and (7) toroidal field; (8) fraction of the poloidal field which is dipole and (9) symmetric; (10) fraction of the toroidal field which is dipole and (11) symmetric. In columns 12 to 14 there are characteristics of the dipole component: (12) field strength; (13) inclination with respect to the rotation axis in degrees; (14) dipole's pole phase. Column 15 yields the $R V$ value of the system calculated with ZDI.

Since the set of data for December 2008 has a good phase coverage, the obtained parameters, which characterize the magnetic field topology at this epoch, have a very low reliability and have not been used for subsequent analysis and discussion. As can be seen from Table 2, in different observational seasons the degree of surface coverage by dark and bright regions varied from 15 to $18 \%$, whereas the magnetic field strength achieved the values of $0.5-0.6 \mathrm{kG}$. Since the ZDI method is sensitive to only middle- and large-scale surface elements and returns the solution taking into account the maximum entropy, such a small degree of coverage by spots do not contradict our assumption that up to $30 \%$ of the stellar surface can be more or less covered by small dark and light formations.

Similar to brightness maps, the magnetic maps show a complex magnetic field topology with variable poloidal and toroidal components. The poloidal component has a weak dipole constituent and is quite nonaxisymmetric (see Columns 8 and 9 in Table 2). The toroidal component of the field contains up to 45-60\% of the total magnetic energy in 2009-2013 and reduces its contribution up to 20-30\% in 2015-2016. Contrary to the poloidal component, the toroidal component is unusually strong and axisymmetric. The location of the dipole constituent's pole with respect to the stellar rotation axis varies significantly from season to season in the range from 18 to $54^{\circ}$. Meanwhile, the pole's phase is quite stable and lies in the range $0.5-0.6$, whereas the dipole component's intensity gradually grows from $165 \mathrm{G}$ in January 2009 to $~ 400 \mathrm{G}$ in $2015-2016$.

\section{Search for exoplanets}

After using the ZDI method for reconstructing brightness maps of young stars of our sample, we tried to find signatures for the existence of exoplanets in spectral data. Radial velocity (RV) values defined from input spectra as momenta of the first order in a subtraction of Stokes $I$ LSD profiles from the continuum involve the contribution from photospheric inhomogeneities (cool spots and hot areas), which are to be called the stellar activity variations. Reconstructing brightness distribution over the stellar surface using the ZDI method, we practically perform a filtration of stellar activity variations and acquire information concerning relatively actual RV values of a star and can implement a search for the 


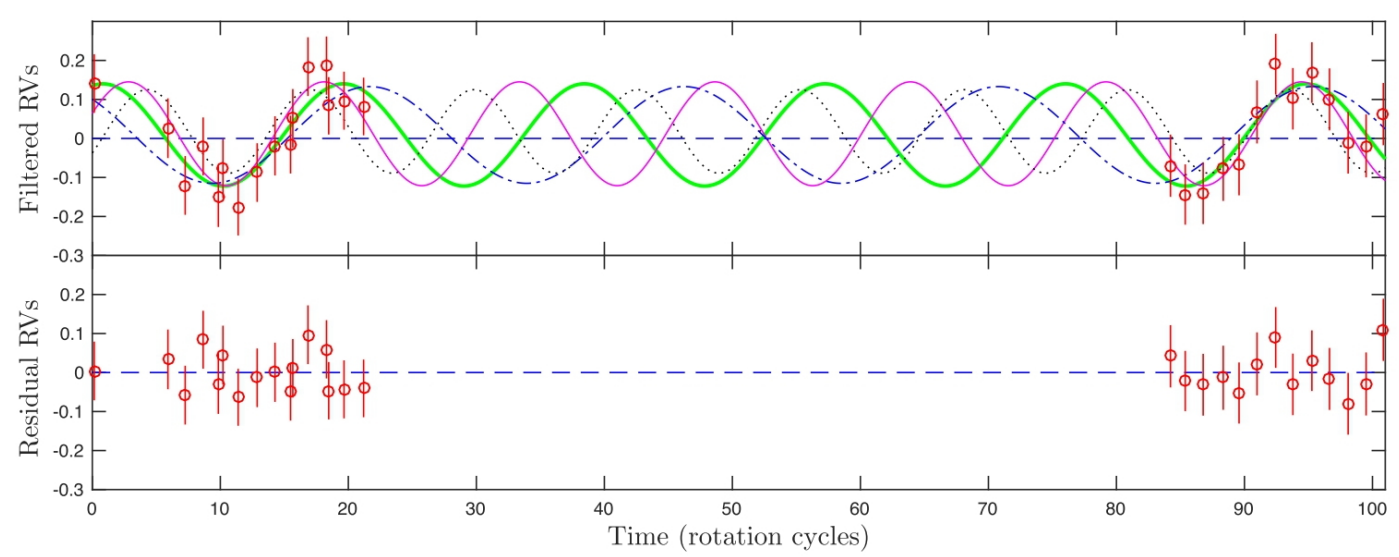

Fig. 7. The filtered RV values for TAP 26 (top panel) and residual RV after extracting the planetary signal (bottom panel). The RV values are given in $\mathrm{km} \mathrm{s}^{-1}$. Details are in the text (Fig. 9 from the paper of Yu et al., 2017)
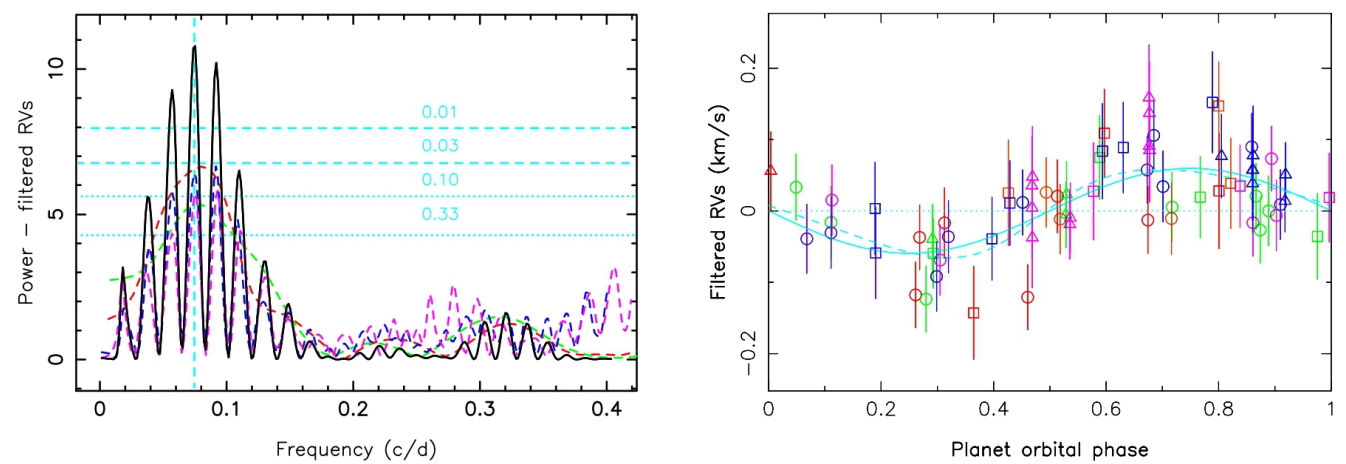

Fig. 8. Results of the periodogram analysis of RV data for TAP 26 (left) and the phase curve of the filtered RV of V830 Tau for $P_{\text {or }}=4.93$ days (right). The left panel shows color periodograms constructed for the whole data set (black line), for November 2015 (red), for January 2016 (green), for all odd data (blue) and for all even data (purple). Levels of false alarm are marked by horizontal lines. The peak that corresponds to $P_{\text {orb }}=10.8$ days is denoted by the vertical dashed line. The right panel with different colors and different signs shows the RV values which correspond to different seasons and different rotations cycles (Fig. 10 from the paper of Yu et al., 2017 and Fig. 8 from the paper of Donati et al., 2017)

signatures of the existence of a planet in these filtered RV values.

With the aim of searching for a signal from a planet we analyzed the filtered RV values using the Lomb-Scargle periodogram for all WTTS from our sample. The periodogram analysis was carried out for both each season separately and the whole data set as a unified time series if a star was observed during several seasons. In most cases, in the time series of the filtered RV values we have not found any reliable periods with possibility of a false alarm lower than $5 \%$. Nonetheless, for two objects, TAP 26 and V830 Tau, we succeeded in detecting evident signatures for the existence of a signal from the planet in the filtered RV values.

As an example, in Fig. 7 we represent a time series of the filtered RV values for TAP 26 before and after deriving a signal from the planet. Four different sinusoids in the top panel of Fig. 7 exhibit several fittings which correspond to different test orbital periods of the planet: the green solid line - for $P_{\text {orb }}=13.4$ days, the purple solid line - for 10.8 days, the blue dash-dotted line - for 17.5 days, and the black dotted line - for 9.1 days. According to the results of the periodogram analysis, the orbital period $P_{\text {or }}=10.79$ days has the highest reliability (see Fig. 8, left panel). Using the most probable approach and assuming that the planet rotates in the equatorial plane of the star, we obtain that the planet TAP 26b has the minimum mass $M \sin i=1.66 \pm 0.31 M_{J u p}$ and located at the distance $0.0968 \pm 0.0032$ a.u. from the star.

In the case of V830 Tau, in the set of the filtered RV data we detected a reliable sinusoidal signal with a semi-amplitude of $68 \pm 11 \mathrm{~m} \mathrm{~s}^{-1}$ which corresponds to $P_{\text {orb }}=4.93$ days (see Fig 8, right panel). Using this period and basic parameters of the central star, we obtain that the V830 Tau b planet has the minimum mass $M \sin i=0.57 \pm 0.10 M_{J u p}$ and located at the distance $0.057 \pm 0.001$ a.u. from the star.

The properties of planets detected in young systems V830 Tau and TAP 26 are better interpreted within the framework 

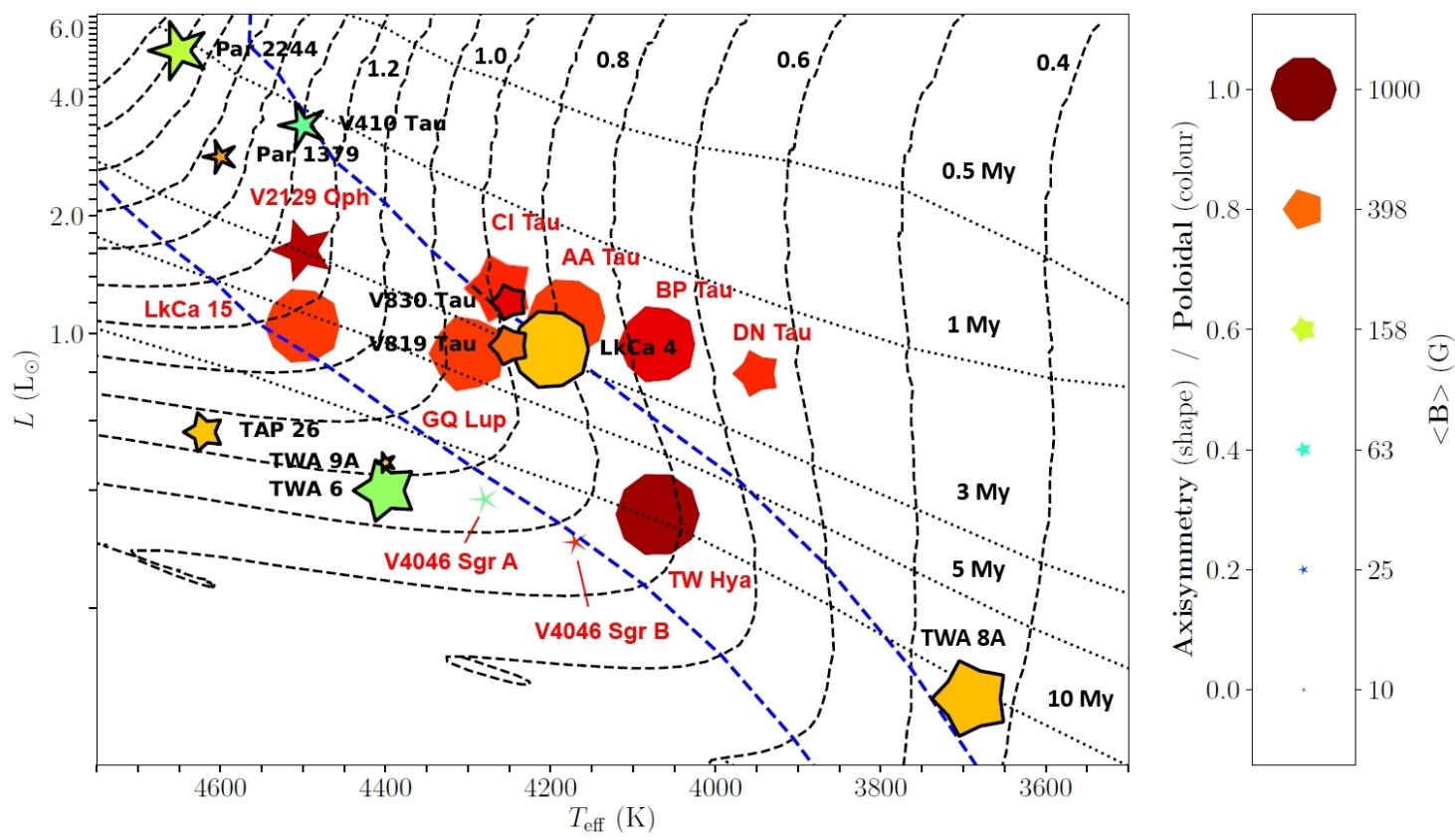

Fig. 9. HR diagram of WTTS within MaTYSSE (names are colored with black) and CTTS within MaPP (names are colored with red). The size of symbols represents the mean magnetic field strength. The color of symbols depends on the contribution of the poloidal component (brown color corresponds to the fully poloidal field). The shape of symbols corresponds to the symmetry of the poloidal component (the high symmetry degree is represented by a symbol that is rounder in shape). Black dashed lines - evolutionary tracks (from 0.4 to $1.9 M_{\odot}$ ), black dotted lines - isochrones (for an age of $0.5,1,3,5$, and 10 million years). Two blue dashed lines show a fraction of the convective envelope on the stellar radius $(100$ and $50 \%)$

of the theory of type II disk migration rather than by the model of planetary scattering with tidal circularization. As compared to the young WTTS V830 Tau with an age of 2 million years, TAP 26 with an age of $\simeq 17$ million years and the analogous mass looks like a more evolved star that rotates 4 times faster than V830 Tau, probably, due to its 4 times lower inertia momentum (according to modern models of evolution). Most likely, its planet TAP 26b finished its migration inside the disk at the current orbital distance (0.097 a.u.) when TAP 26 was young and fully convective star with a strong dipole magnetic field component of several $\mathrm{kG}$, which could destroy the disk at the distance 0.09 a.u. This destruction of the disk occurred quite rapidly when the star had an age of about 1-2 million years, i.e., before the large-scale magnetic field became weaker with a more complex topology.

We can assume that the younger planet V830 Tau b, whose orbital period is longer than the rotation period of the central star, will migrate until the star spins down. The question as to whether the tidal forces will have been strong enough by that time to replace the planet closer to the star and provoke its falling in a few hundreds of millions of years remains open.

\section{Conclusions}

As a result of implementing two international research programs MaPP and MaTYSSE, the numerous photometric and spectropolarimetric data for eight TTS in the Taurus - Auriga star-forming region were acquired. An analysis of these data using the tomographic ZDI method made it possible to reconstruct the brightness distribution over the stellar surface and reconstruct the large-scale magnetic field topology of TTS.

Two CTTS (AA Tau and LkCa 15) were shown to have axisymmetric poloidal magnetic fields with a strong dipole component and are in the propeller mode when the rotation of the young star decelerates due to a magnetic relation between the star and protoplanetary disk. The third CTTS (V2129 Oph) demonstrates a strong poloidal field that is not axisymmetric, whereas the octupole component is 2-3 times stronger than the dipole. Moreover, it was detected that the magnetic field of this star is nonstationary on a time scale of a few years. A variation of magnetic field properties was revealed for WTTS V410 Tau. The intensity of the dipole magnetic field component was shown to monotonically increase from 165 to $458 \mathrm{G}$ throughout 6 years. Meanwhile, the contribution of the dipole component into the poloidal field varied in the range from 23 to $38 \%$.

An analysis of the filtered RV values of five WTTS made it possible to first detect signatures for the existence of giant exoplanets for TAP 26 and V830 Tau with masses of an order of 1.7 and $0.6 M_{J u p}$, respectively. These planets are located at the distances of about 0.097 and 0.057 a.u. from TAP 26 and V830 Tau, respectively, which is in a good agreement with the theory of type II disk migration.

Five WTTS studied in our sample exhibit a wider range of magnetic field topologies as compared to the magnetic field topology of three CTTS. To be sure in these prelimi- 
nary conclusions, the data on magnetic fields using a more extensive sample of young stars is required. With this aim, we invoked information on the magnetic field topology of all the TTS studied up to date, which is available in recent publications (see, e.g., Hill et al., 2019). Figure 9 shows the HR diagram with magnetic field topologies of ten CTTS and ten WTTS studied using the ZDI method within the MaPP and MaTYSSE programs. This figure was constructed based on our initial graphs which were used in the first rationales of MaPP and MaTYSSE programs in 2013-2015 and subsequently published several times by the MaTYSSE group in various versions. In particular, one of the recent versions was given in the paper of Hill et al. (2019). The current Fig. 9 was complemented with new data on the magnetic field topology of CI Tau. In this figure, for ease of reading, the names of stars were superimposed, the evolutionary tracks were signed, and isochrones were marked.

Figure 9 shows that contrary to WTTS from the MaTYSSE project CTTS from the MaPP project exhibit stronger magnetic fields in which the axisymmetric poloidal component dominates. If one considers the sample of WTTS and CTTS as a single group of TTS, then it can be noted that the magnetic field is generally poloidal and axisymmetric on cooler (lower than $4300 \mathrm{~K}$ ) young stars which remain to a great extent convective. Hotter (more massive) TTS have less poloidal and less axisymmetric magnetic fields independent of the degree of development of the radial core and sizes of the outer convective envelope (V2129 Oph, V410 Tau, Par 1379, and Par 2244). These conclusions should be confirmed based on a more comprehensive sample of young stars.

We emphasize importance of studying large-scale magnetic fields for a more comprehensive sample of stars with the aim of testing and developing stellar dynamo models and theories of formation of solar-type stars and their planets. Spectropolarimeters-velocimeters of the new generation operating in the near infrared range such as SPIRou (Artigau et al., 2014) capable of providing access to younger stars and having high sensitivity to magnetic fields and radial velocities should be the main active for successive solving the above tasks.

Acknowledgements. Authors are grateful to the referee for useful remarks which helped to eliminate a series of shortcomings in the initial version of the paper. The work was supported by the grant of the Government of the Russian Federation and the Ministry of Science and Higher Education of the Russian Federation (\# 075-15-2020-780).

\section{References}

Alencar S.H.P, Bouvier J., Donati J.-F., Alecian E., Folsom C.P., Grankin K., et al., 2018. Astron. Astrophys., vol. 620, id. A195.

Artigau E., Kouach D., Donati J.-F., et al., 2014. Proceedings of the SPIE, vol. 9147, id. 914715.

Grankin K.N., Bouvier J., Herbst W., Melnikov S.Yu., 2008. Astron. Astrophys. vol. 479, pp. 827-843.

Grankin K.N., Artemenko S.A., 2009. Inf. Bull. Var. Stars, no. 5907 , pp. $1-4$.

Grankin K.N., 2013. Astron. Lett., vol. 39, no. 7, pp. 01-12.

Donati J.-F., Jardine M.M., Gregory S.G., Petit P., Bouvier J., et al., 2007. Mon. Not. Roy. Astron. Soc., vol. 380, pp. 1297-1312.

Donati J.-F., Skelly M.B., Bouvier J., Gregory S.G., Grankin K.N., et al., 2010. Mon. Not. Roy. Astron. Soc., vol. 409, pp. 1347-1361.

Donati J.-F., Bouvier J., Walter F.M., Gregory S.G., Skelly M.B., et al., 2011. Mon. Not. Roy. Astron. Soc., vol. 412, pp. 2454-2468.

Donati J.-F., Hébrard E., Hussain G., Moutou C., Grankin K., et al., 2014. Mon. Not. Roy. Astron. Soc., vol. 444, pp. 3220-3229.

Donati J.-F., Hébrard E., Hussain G.A.J., Moutou C., Malo L., Grankin K., et al., 2015. Mon. Not. Roy. Astron. Soc., vol. 453, pp. 3706-3719.

Donati J.-F., Yu L., Moutou C., Cameron A.C., Malo L., Grankin K., et al., 2017. Mon. Not. Roy. Astron. Soc., vol. 465, pp. 3343-3360.

Donati J.-F., Bouvier J., Alencar S.H., Hill C., Carmona A., Folsom C.P., et al., 2019. Mon. Not. Roy. Astron. Soc., vol. 483, pp. L1-L5.

Morin J., Donati J.-F., Petit P., Delfosse X., Forveille T., et al., 2008. Mon. Not. Roy. Astron. Soc., vol. 390, pp. 567-581.

Skelly M.B., Donati J.-F., Bouvier J., et al., 2010. Mon. Not. Roy. Astron. Soc., vol. 403, pp. 159-169.

Hill C.A., Folsom C.P., Donati J.-F., et al., 2019. Mon. Not. Roy. Astron. Soc., vol. 484, pp. 5810-5833.

Yu L., Donati J.-F., Hébrard E., Moutou C., Malo L., Grankin K., et al., 2017. Mon. Not. Roy. Astron. Soc., vol. 467, pp. 1342-1359.

Yu L., Donati J.-F., Grankin K., et al., 2019. Mon. Not. Roy. Astron. Soc., vol. 489, pp. 5556-5572. 